\title{
Erratum
}

\section{Erratum to: An Optimization Approach to Inverse Dynamics Provides Insight as to the Function of the Biarticular Muscles During Vertical Jumping}

\author{
Daniel J. Cleather, ${ }^{1,2}$ Jon E. Goodwin, ${ }^{1}$ and Anthony M. J. Bull ${ }^{2}$ \\ ${ }^{1}$ St. Mary's University College, Waldegrave Road, Twickenham TW1 4SX, UK; and ${ }^{2}$ Department of Bioengineering, \\ Imperial College London, London, UK
}

\section{Erratum to: Annals of Biomedical Engineering (2011) 39(1):147-160 DOI 10.1007/s10439-010-0161-9}

The authors regret that equations of motion given in Eq. (6) of Ann Biomed Eng, 2011; 39:147-1601 did not include the moment created by the joint contact forces arising due to the action of the biarticular muscles. ${ }^{2}$ The correct equation should read: analytically that the notional inter-segmental joint moment is equivalent to the traditional approach.

Given the notional inter-segmental joint moments are equivalent between all the cases presented in the revised analysis, the activation of the musculature is more constrained than in the original paper. In particular, this results in the activity of the biarticular muscles being generally $0.2-0.6 \times \mathrm{BW}$ lower than in

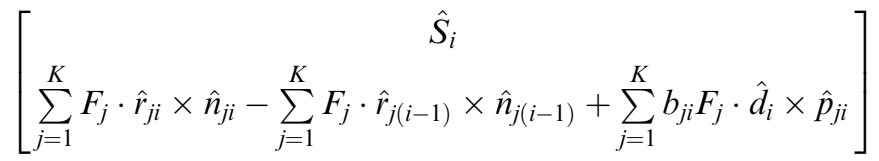

$$
\begin{aligned}
& =\left[\begin{array}{cc}
m_{i} E_{3 \times 3} & 0_{3 \times 3} \\
m_{i} \tilde{c}_{i} & I_{i}
\end{array}\right]\left[\begin{array}{c}
\hat{a}_{i}-\hat{g} \\
\ddot{\hat{\theta}}_{i}
\end{array}\right]+\left[\begin{array}{c}
0_{3 \times 1} \\
\dot{\hat{\theta}}_{i} \times I_{i} \dot{\hat{\theta}}_{i}
\end{array}\right]+\left[\begin{array}{cc}
E_{3 \times 3} & 0_{3 \times 3} \\
\tilde{d}_{i} & E_{3 \times 3}
\end{array}\right]\left[\begin{array}{c}
\hat{S}_{i-1} \\
\hat{M}_{i-1}
\end{array}\right]
\end{aligned}
$$

where $b_{j i}=1$ for biarticular muscles that cross but do not attach to segment $i ; b_{j i}=0$ for all other muscles and $\hat{p}_{j i}=$ line of action of biarticular muscle $j$ about segment $i$.

Figure 2 should additionally be replaced by the figure below (incorporating definitions of the new quantities in Eq. (6)).

The authors had previously demonstrated that this approach to the inverse dynamics problem permitted the notional inter-segmental joint moment to exceed that calculated by a traditional inverse dynamics analysis. However, when the joint contact force due to the biarticular muscles is included, it can be shown

Address correspondence to Daniel J. Cleather, St. Mary's University College, Waldegrave Road, Twickenham TW1 4SX, UK. Electronic mail: dancleather@hotmail.com

The online version of the original article can be found under doi: 10.1007/s10439-010-0161-9. the original paper. This also results in a commensurate decrease in the calculated joint reaction forces, but these differences are generally small. The largest decreases are seen at the hip and at the PFJ during landing with the largest being seen at the hip during landing $(1.2 \times \mathrm{BW}-17 \%)$.

The temporal pattern of the activation of biarticular muscles in the revised BI and BIH approaches showed much more similarity to the TRADB approach, but there were still differences. An excerpt from Figure 4 is reproduced here to exemplify this.

The difference between the TRADB and BI methods is due to the formalization of different optimization problems. On the one hand the TRADB method is based purely upon a consideration of joint moments, where each biarticular muscle is modeled as two monoarticular muscles that are constrained to have the same force. In contrast the BI approach is based upon segmental considerations. This permits a more realistic representation 

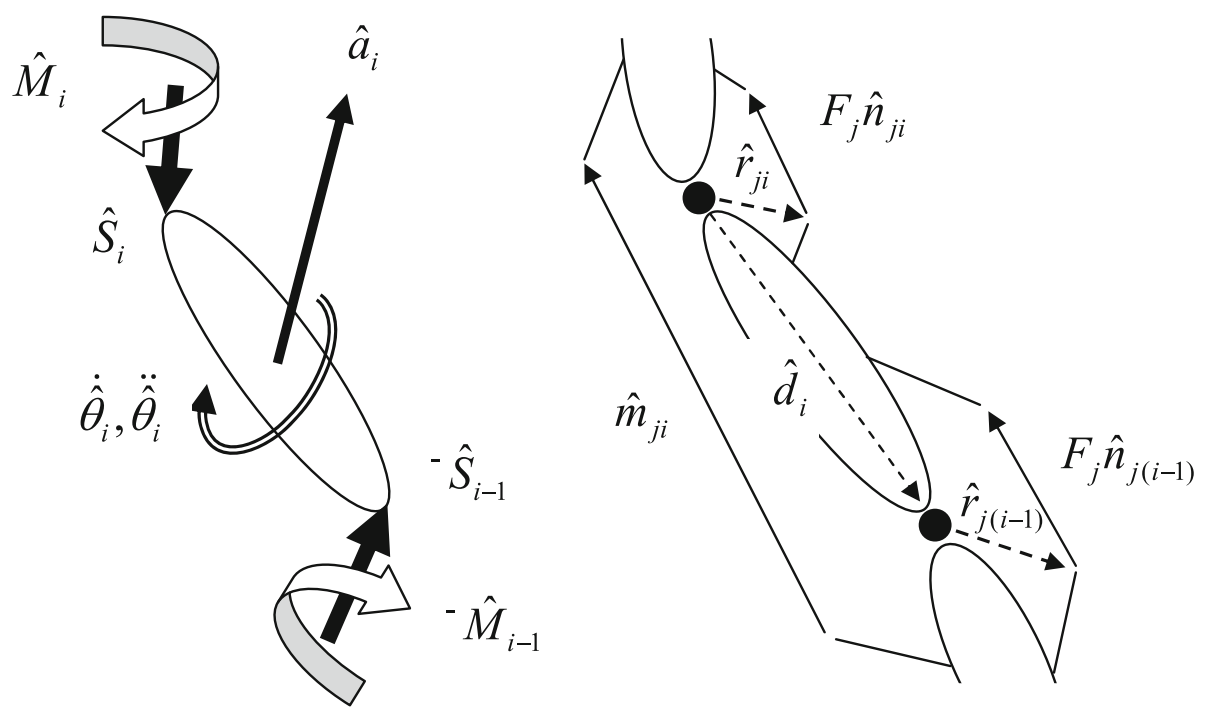

Segmental Variables

\section{Muscular Variables}

FIGURE 2. Nomenclature used in the 1- and 2-step approaches for segment $i$.

TRADB

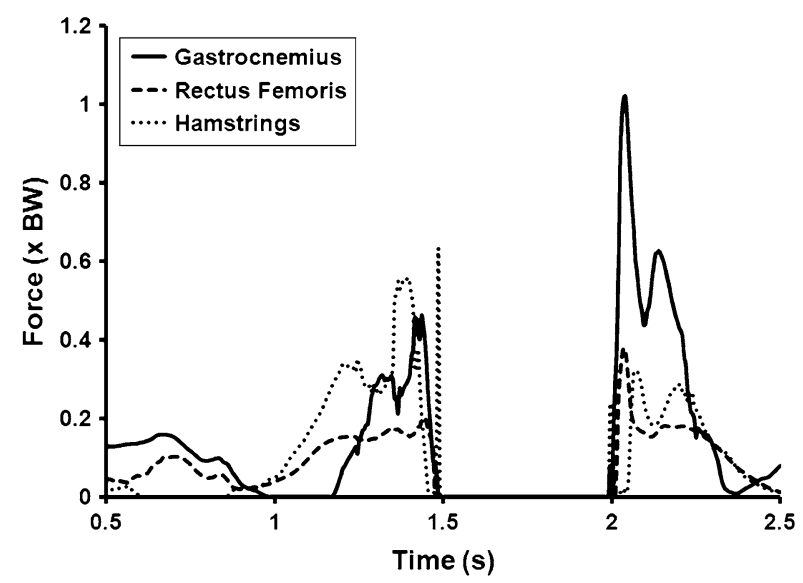

BI

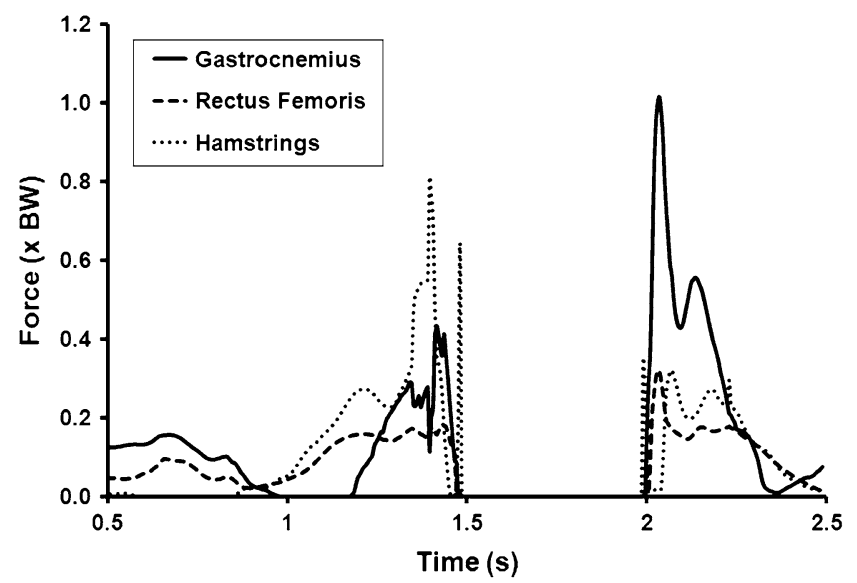

FIGURE 4. (excerpt). Selected biarticular muscle forces for a typical subject (Subject 12) based on the TRADB and BI methods.

of the biarticular muscles. Figure A1 contrasts the two approaches to modeling a biarticular muscle. Although it can be shown that the net moment acting on the intermediate segment is equivalent between the two approaches, it is clear that the moments impressed at either end of the intermediate segment are different.

During vertical jumping it is common for a biarticular muscle to be an agonist at one joint and an antagonist at the other, which, when modelled in this way under the traditional approach, constrains its activity. Alternatively, in the segment based approach the biarticular muscles cause the rotation of three segments, and during vertical jumping can often act to assist the rotation of two segments while impeding the other. This difference in the optimization problem thus permits the difference in activity of the biarticular muscles between the two approaches.

In summary, if the effect of the joint contact forces due to the biarticular muscles is included in Eq. (6), the TRADB and BI cases are more similar than previously 

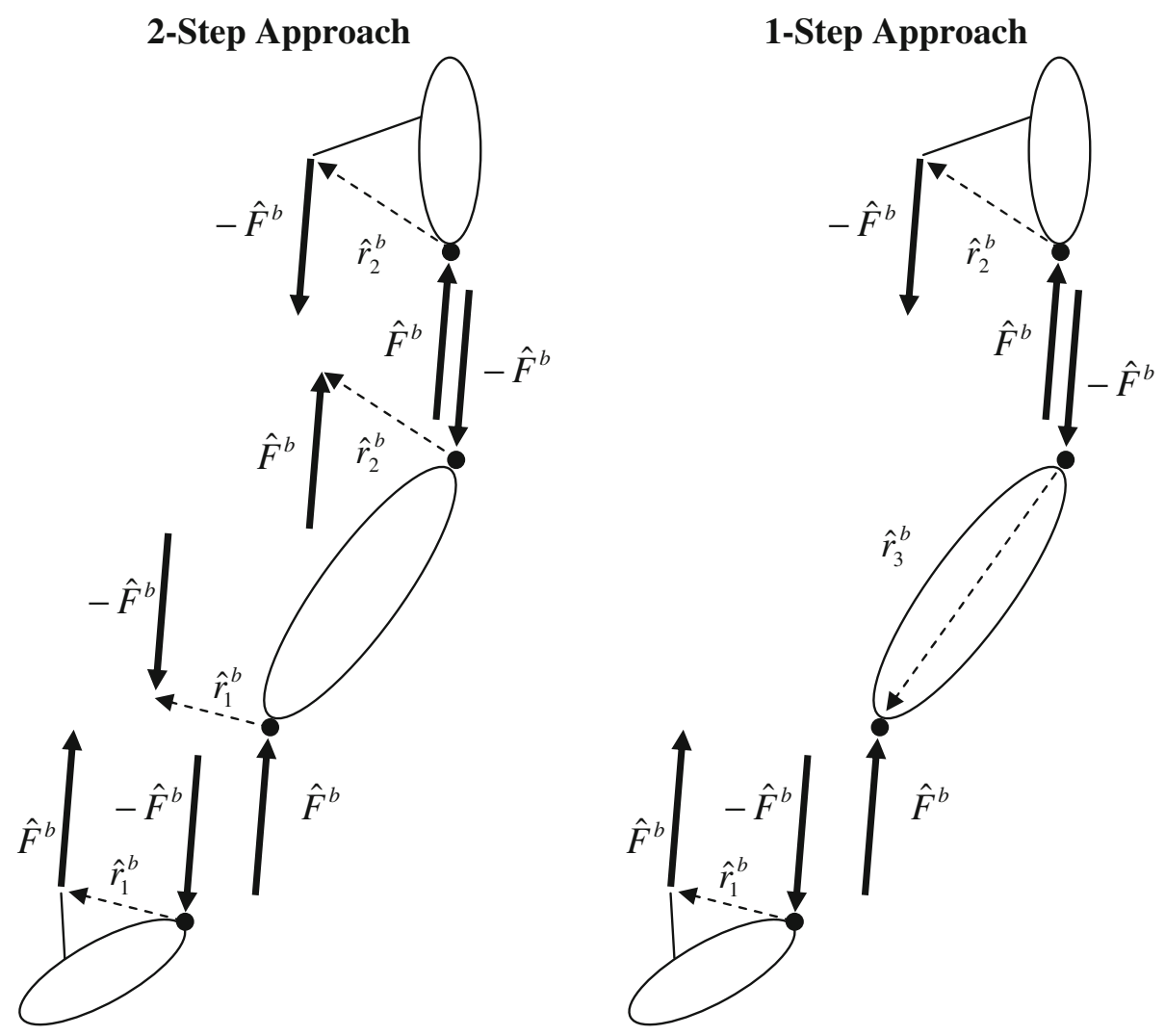

FIGURE A1. Modelling biarticular muscles in the 1- and 2-step approaches.

presented. Despite this there are still differences between the two cases due to the formalization of different optimization problems. The authors therefore suggest that the method presented here represents a more faithful interpretation of the biarticular muscles and should therefore be preferred in future work.

The authors would like to thank Frans van der Helm for pointing out the error in the original paper.

\section{REFERENCES}

${ }^{1}$ Cleather, D. J., J. E. Goodwin, and A. M. J. Bull. An optimization approach to inverse dynamics provides insight as to the function of the biarticular muscles during vertical jumping. Ann. Biomed. Eng. 39:147-160, 2011. doi:10.1007/ s10439-010-0161-9.

${ }^{2}$ Zatsiorsky, V. M. Kinetics of Human Motion. Champaign, IL: Human Kinetics, 672 pp., 2002. 